\title{
Straw tube studies and prototype Assembly
}

*Bhumika Mehta, 'Daisy Kalra, Jyoti Tripathi, *Prachi Sharma, Vipin Bhatnagar Department of Physics, Panjab University, India

${ }^{1}$ Columbia University, New York

\section{Introduction}

Straw tube detector is a gaseous drift detector in which each thin proportional tube (straw) acts as a single channel drift tube. This detector is proposed to being used as a part of one of the configurations of SAND(System for on-Axis Neutrino Detection) in the long baseline neutrino experiment ,DUNE, at Fermilab.

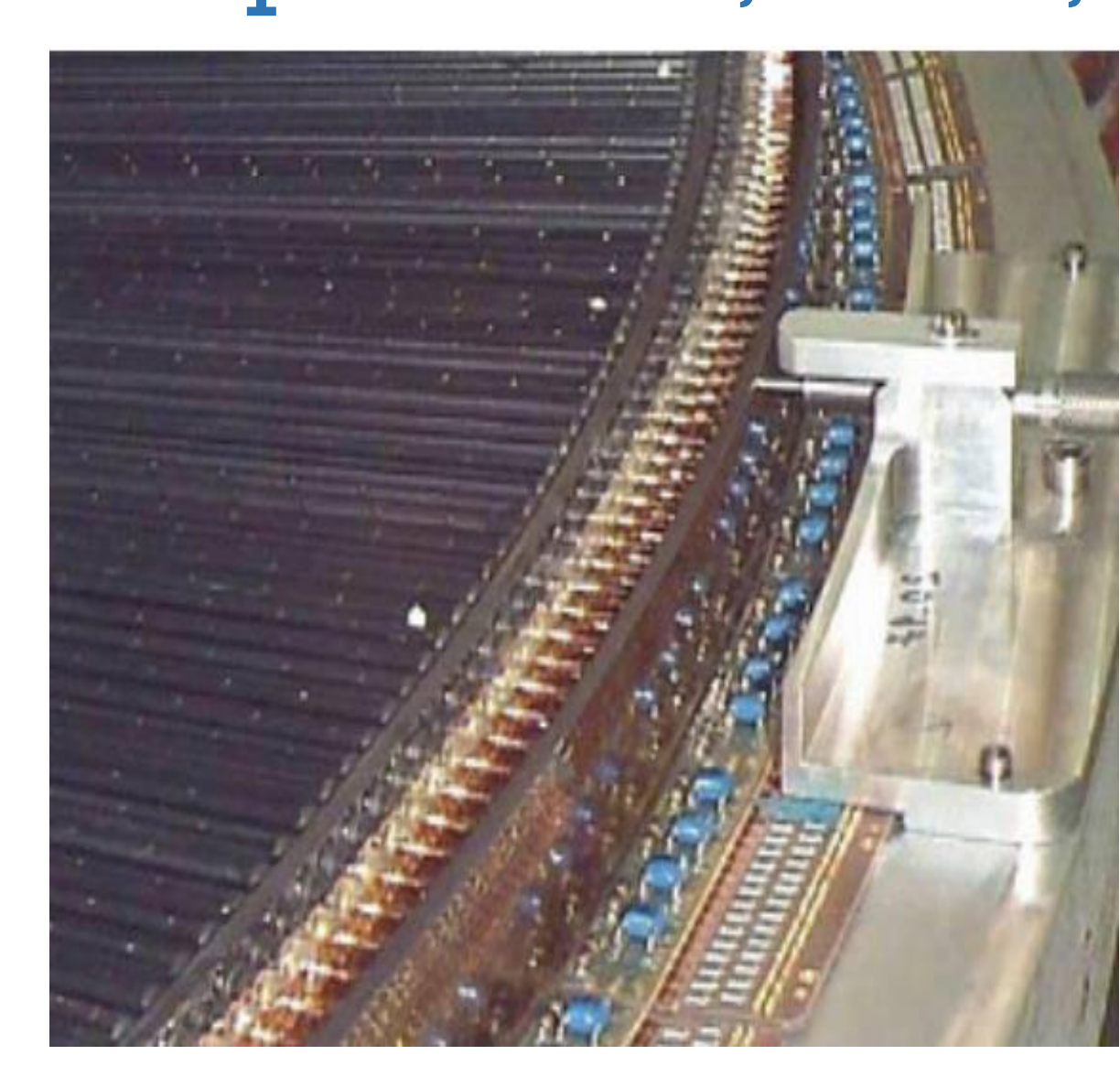

End cap at ATLAS TRT

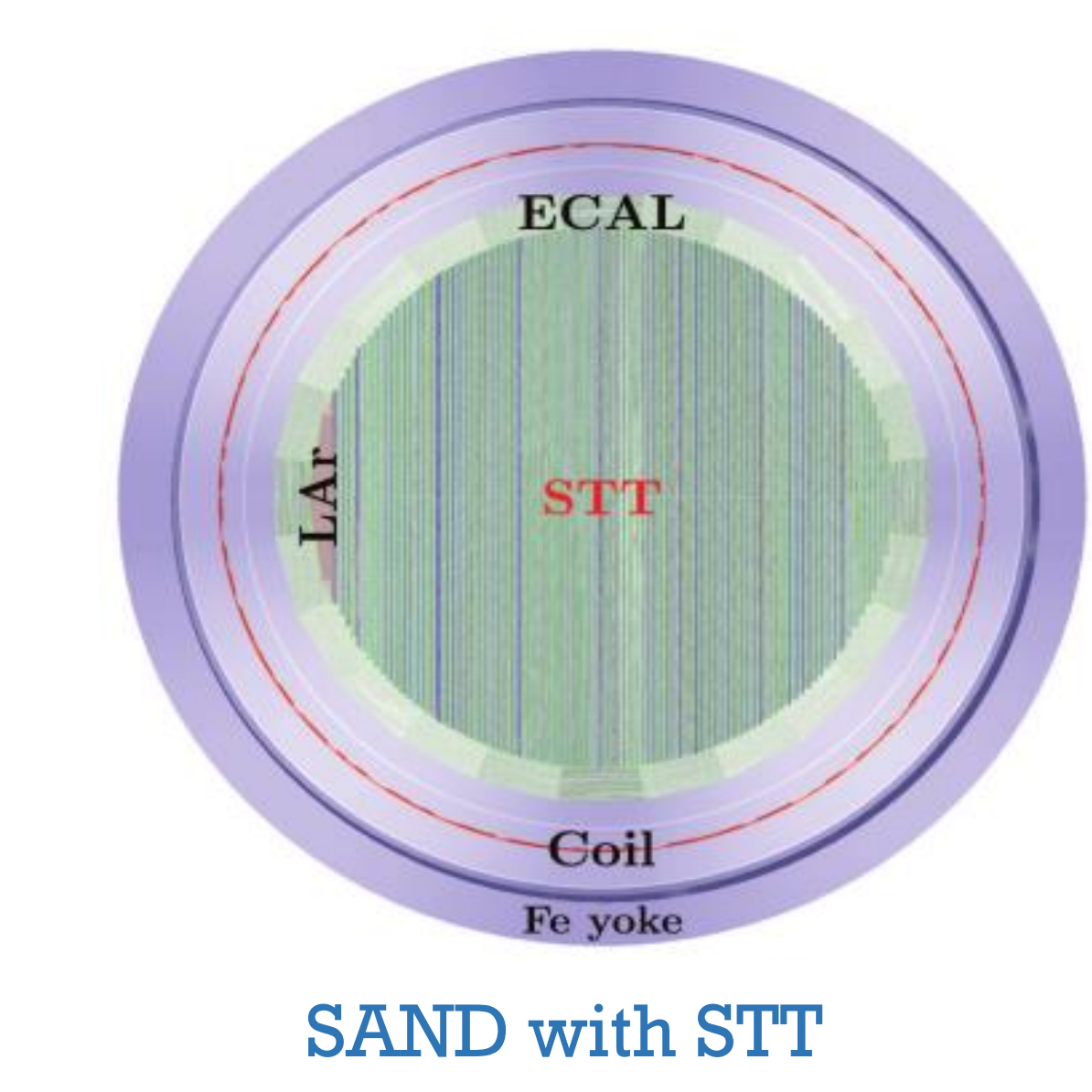

Used in ATLAS, NA62, COMPASS Low cost detector with excellent particle identification

Our group has an experience of working with gaseous detectors like GEM, RPC.We

have 4-channel pneumatic custom-built gas

distribution system, humidifier with

moisture controller for gases in our department.

\section{Test Chamber}

Prototype we are testing is from JINR, Dubna

Kapton Straw tubes with $9.3 \mathrm{~mm}$ diameter 48 Straws in a Single layer

Signal recorded from all 48 straws

Na-22 used as source

Gas mixture: Ar/CO2 (80:20) supplied at 0.3 bar pressure

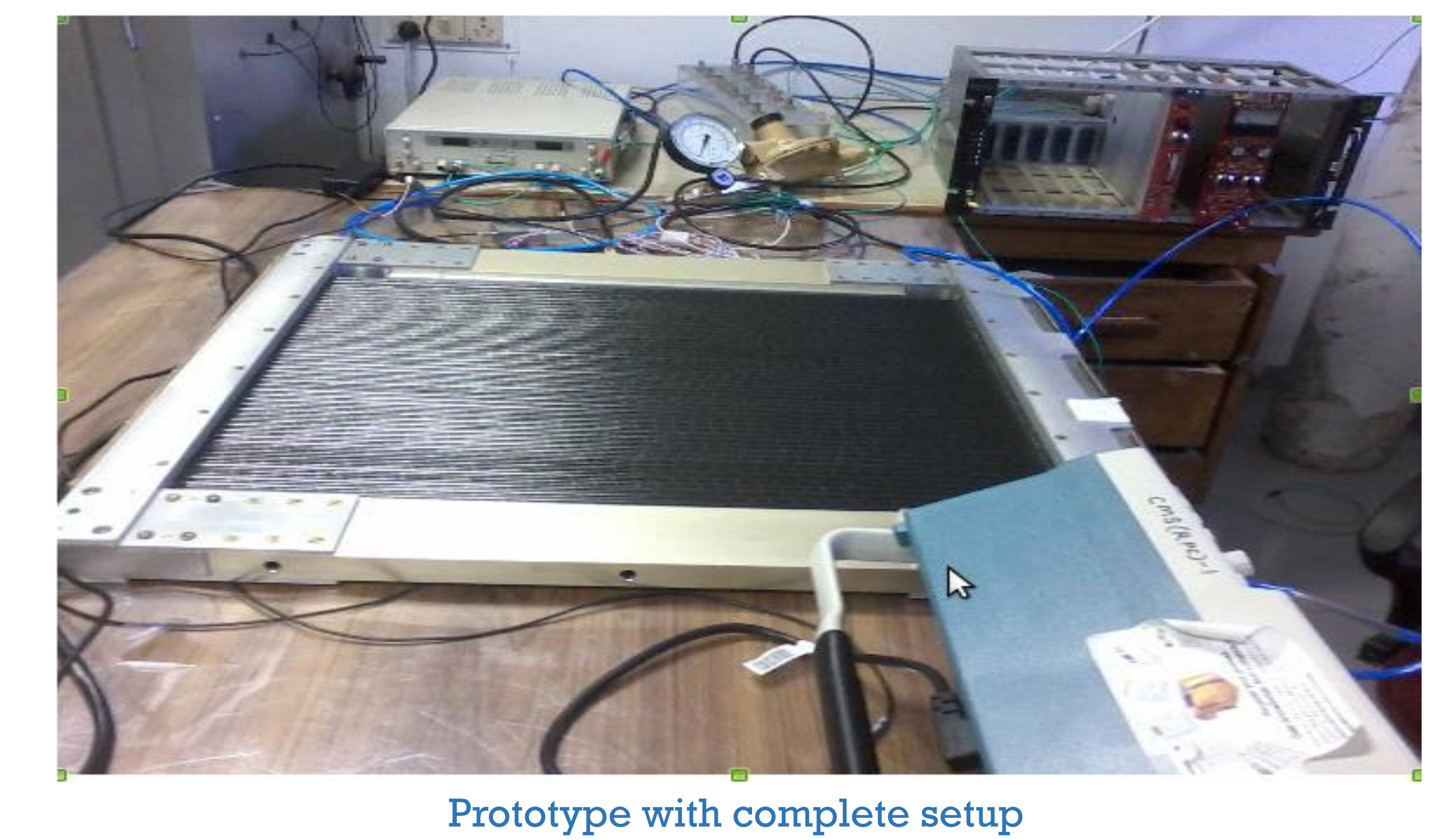

Dual Voltage supply was connected to the preamplifier. Input of pre-amplifier is connected to the channels (to be read out) through

connectors and output is connected to the CRO. High Voltage supply $(1.75-2.00 \mathrm{kV})$ is given to the anode. Whole apparatus was grounded and signal was taken from anode with respect to ground.

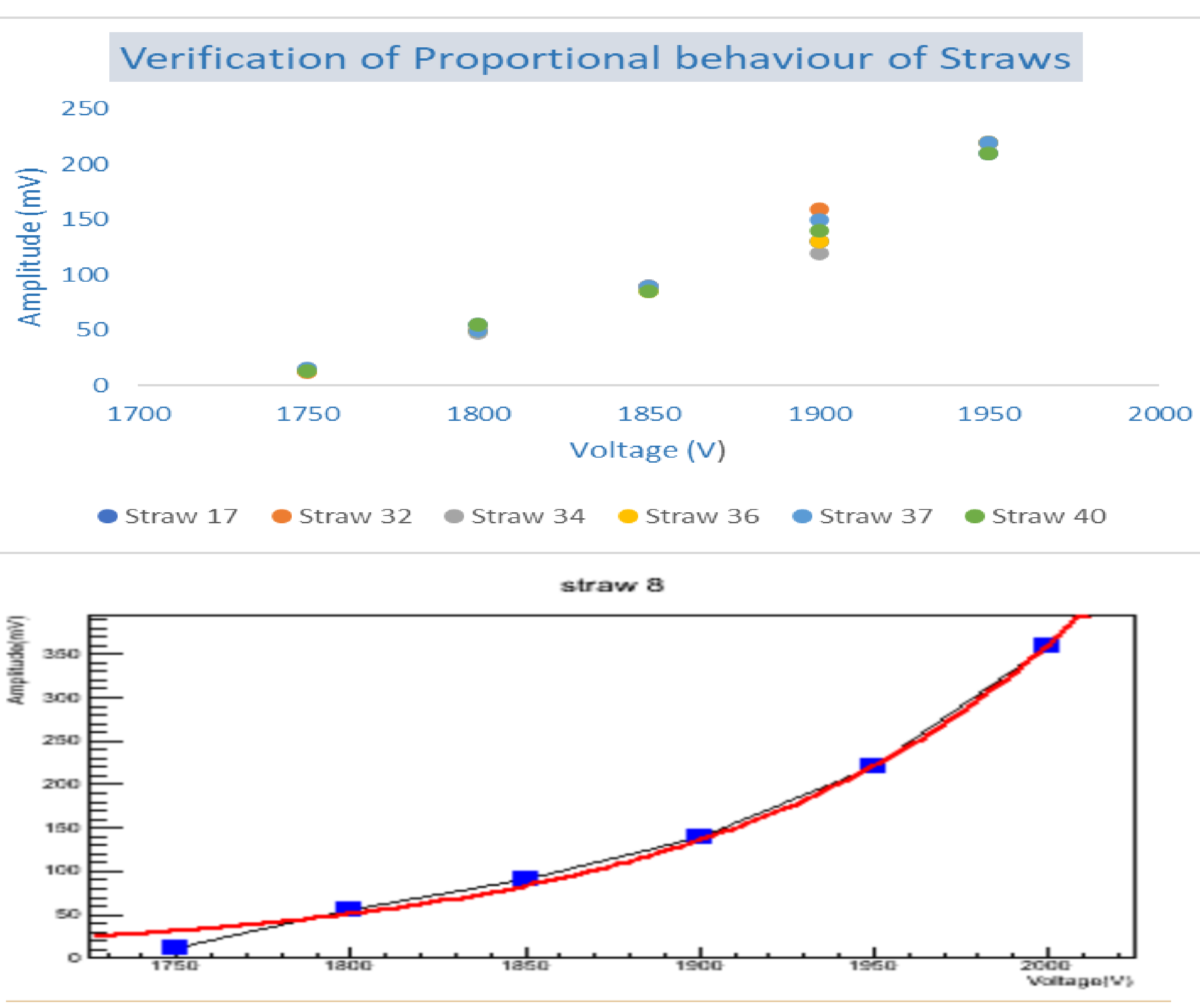

V-A curve for random straw tube (Straw 8)

Black curve represents the experimental values and the red curve is exponentially fitted curve

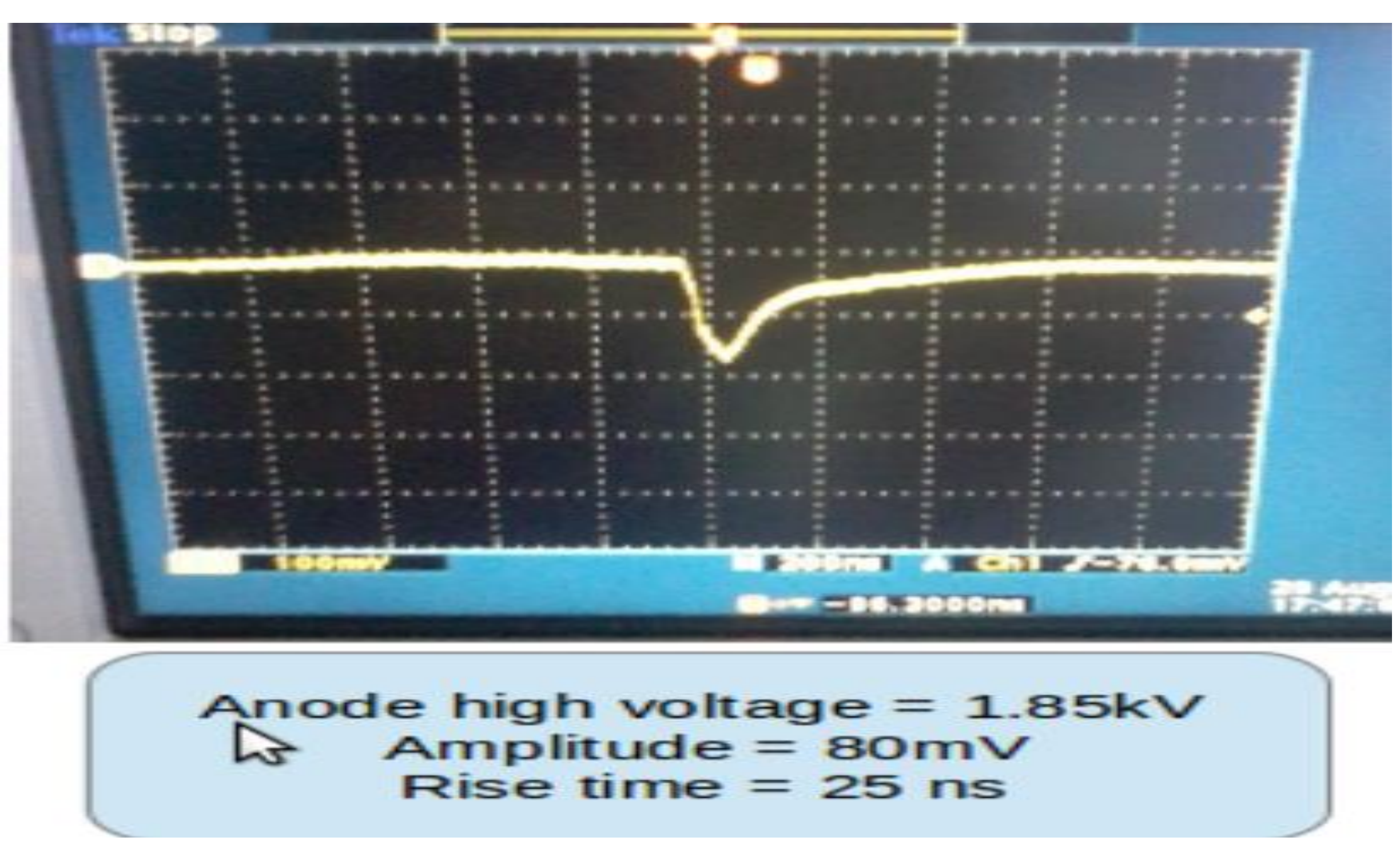

Signal on CRO

\section{ST Assembly and}

Prototyping

Cathode: Mylar tubes

Length: $181 \mathrm{~cm}$

Diameter: $9.5 \mathrm{~mm}$

Anode: Gold plated tungsten wire

Thickness: $20 \mu \mathrm{m}$

Density: $19.22 \mathrm{~g} / \mathrm{cc}$
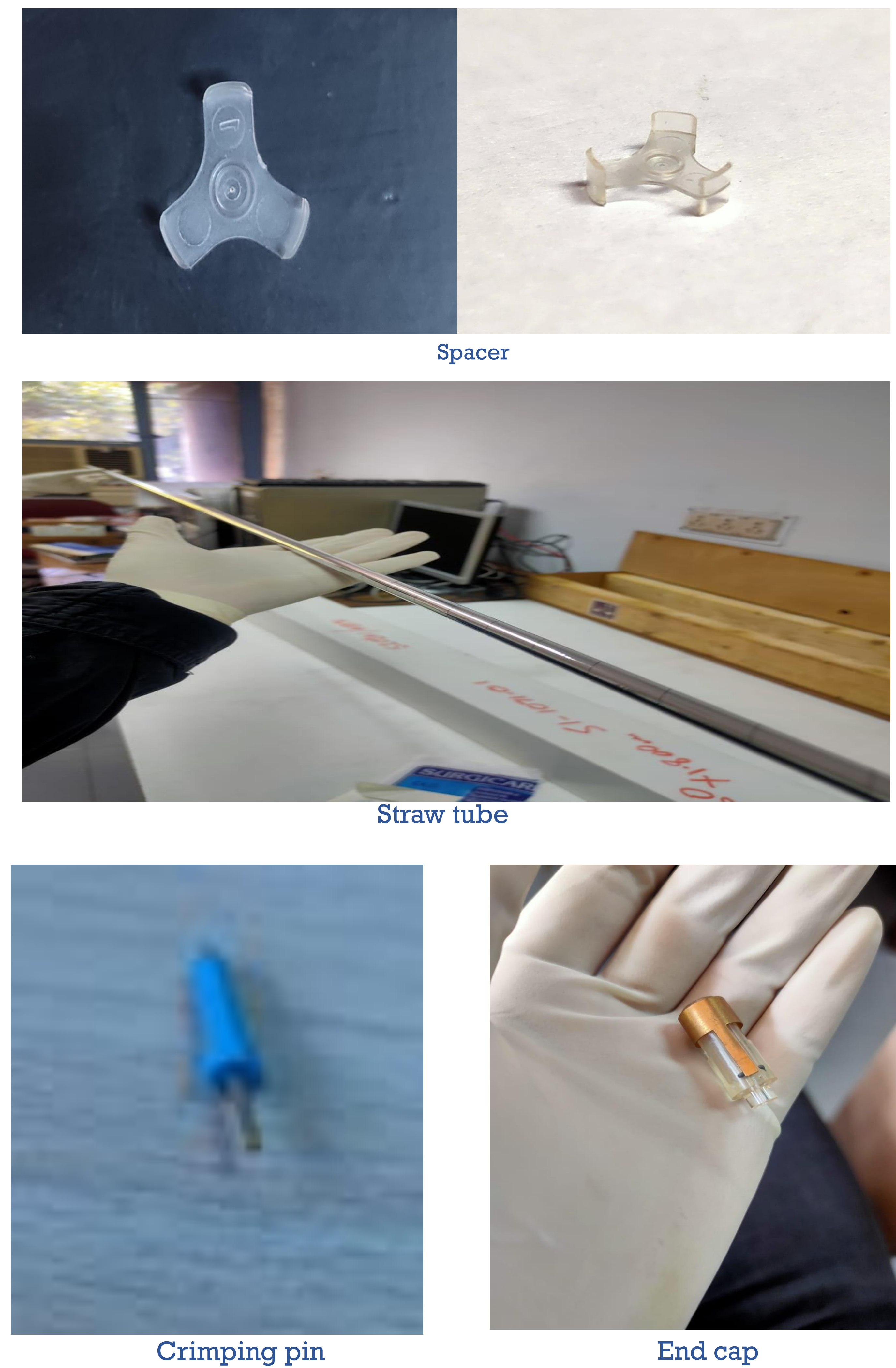

Inserted anode wire in the spacer

Passed through the tube such that the spacer lies in the middle of the straw tube.

End-caps were inserted at both ends. Anode wire was passed through the crimping pin.

Pin was placed inside end-cap (at one end) and crimped. The end-cap was carefully sealed with the help of glue leaving space for the passage of gas.

Tension of $46 \mathrm{gm}$ was given at the other end and the pin was crimped

\section{Perspex chamber was also prepared}

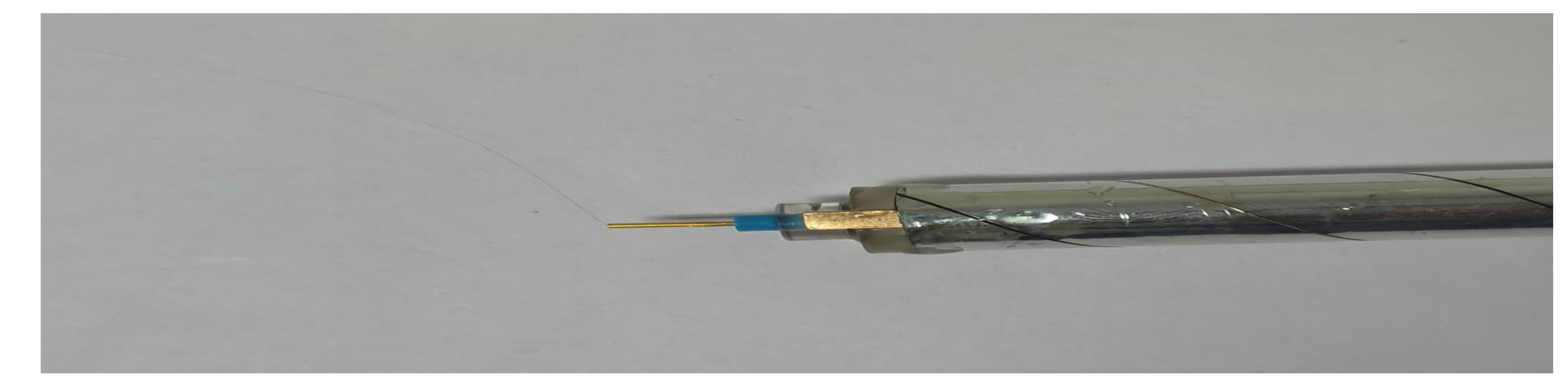

Straw tube after assembly

\section{Future Plans}

Study V-I characteristics of Single straw with pre-mix gas

X-ray images for single spacer effects on the wire

Design a prototype of $1.8 \mathrm{~m}$ X $50 \mathrm{~cm}(\sim 50$ straws)

To explore various tooling ways for assembly of single straw tube

Study with different readout chips

Test the prototype in Cosmic Ray stand

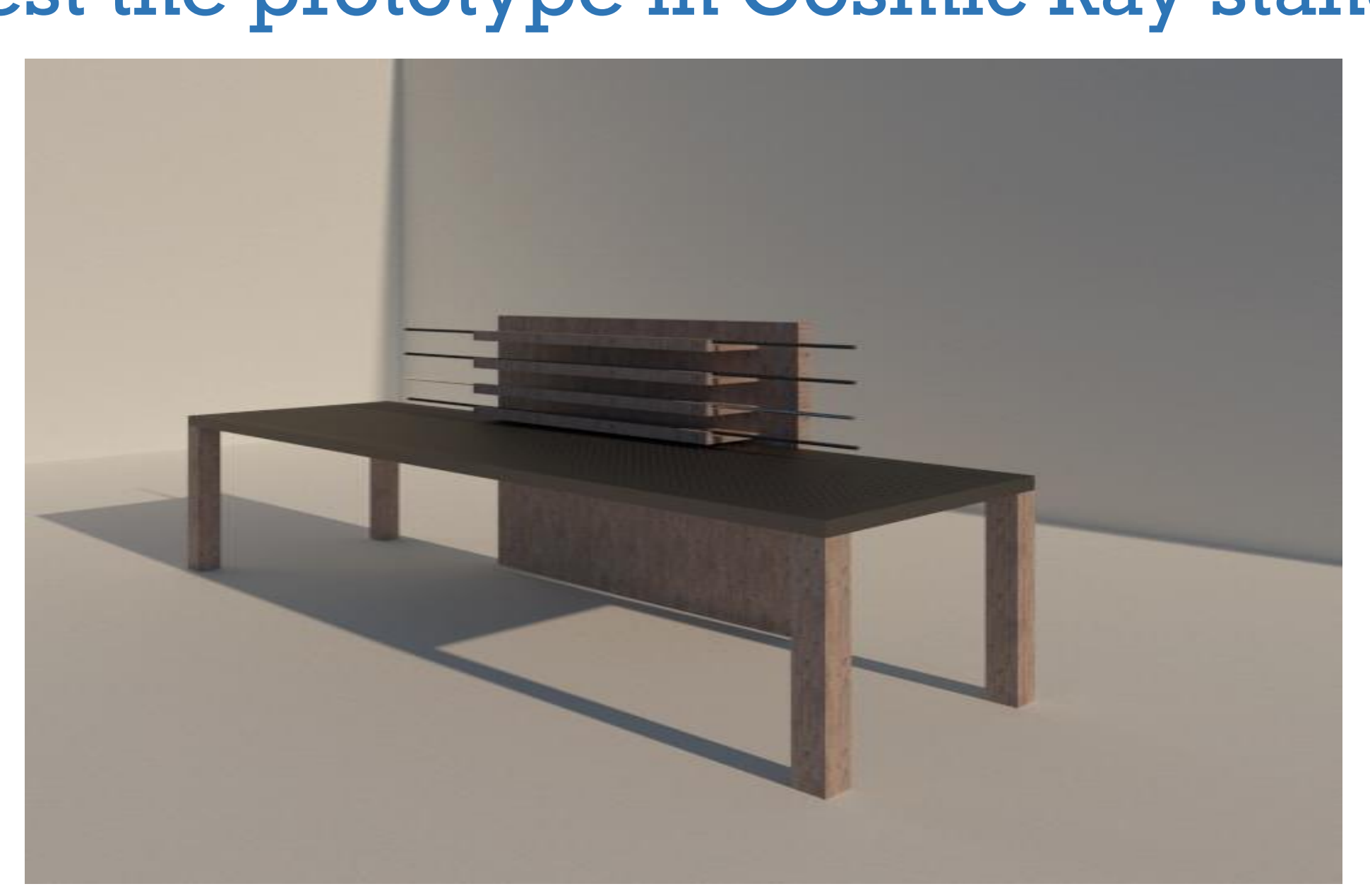

Cosmic ray stand for testing

\section{Acknowledgements}

We acknowledge the enduring support and funding provided by Department of Science and Technology, Government of India.

We are thankful to Riya Gaba(post graduate student) for making a 3D modelled prototype diagram for future studies.

\section{References}

ATLAS Technical Design Report httos://atlas.cern/glossary/tdr NA62 Technical Design Report https://cds.cern.ch/record/1404985 LBNF/DUNE Conceptual Design Report, Volume 4

. LBNE-ND: STT RD AT PU, Vipin Bhatnagar in Neutrino Workshop and Meeting on HEP Instrumentation Center. 